\title{
Cryo-FIB Sample Preparation for Cryo-ET With the Volta Phase Plate
}

\author{
Miroslava Schaffer ${ }^{1}$, Benjamin D. Engel ${ }^{1}$, Marc Wehmer ${ }^{1}$, Sahradha Albert $^{1}$, Eri Sakata ${ }^{1}$, Anna Rast $^{2}$,
} Jörg Nickelsen ${ }^{2}$, Jürgen M. Plitzko ${ }^{1}$ and Wolfgang Baumeister ${ }^{1}$

1. Max Planck Institute of Biochemistry, Department of Molecular Structural Biology, Am Klopferspitz 18, Martinsried, Germany

2. Biozentrum Ludwig-Maximilians-Universität (LMU), Department Biologie I - Botanik, Grosshaderner Strasse 2-4, Martinsried, Germany

Cryo-electron tomography (cryo-ET) of specimens prepared with a cryo-focused ion beam (cryo-FIB) is a powerful technique for in situ studies of cellular structures in their native state [1,2]. Early application of the cryo-FIB to Chlamydomonas reinhardtii cells demonstrated the potential of this approach by unveiling the detailed structure of the chloroplast [3] and revealing novel protein arrays within the Golgi cisternae [4].

By combining the latest generation direct detection cameras with Volta phase plate (VPP) contrast enhancement [5], membrane-bound complexes such as photosystem II and cytochrome b6f were observed in their native environment for the first time [6]. This ability to directly visualize small macromolecules within the cell opens a new frontier for in situ structural biology, where structures of native complexes can be determined, often resolving specific conformational or assembly states. These classified molecular structures can then be mapped back into the cellular volume $[7,8]$.

However, a key prerequisite for successful cryo-ET utilizing the VPP is a specimen of the highest quality, as any negative influences from an insufficient sample can quickly outweigh the contrast benefits of the VPP. To allow visualization and classification of small macromolecules within the cell, the frozen-hydrated specimen must be thin $(<150 \mathrm{~nm})$ and free of both surface contamination and nonvitreous ice. Additionally, the VPP requires that the specimen does not charge during cryo-ET acquisition, a common issue due to the low conductivity of frozen cellular material. To increase the specimen's conductivity, we developed two coating procedures: a simple surface coating by sputtering a very thin platinum layer [7], and a more evolved 'sandwich-technique' that avoids the thin surface coating's effect on image contrast during cryo-ET, but increases sample conductivity only at certain areas [6].

In this work, we will present the practical aspects of cryo-FIB sample preparation for cryo-ET with VPP imaging. We will showcase the application of this technique to the investigation of various macromolecules, including the $26 \mathrm{~S}$ proteasome (Figure 1) and membrane-bound photosynthetic complexes. Data from different organisms will be compared to highlight specimen-dependent influences on data acquisition and analysis. [9] 
References:

[1] A Rigort et al, PNAS 109(12) (2012), p. 4449.

[2] M Schaffer et al, Bio-protocol 5(17) (2015), e1575.

[3] B Engel et al, eLife (2015), 4:e04889.

[4] B Engel et al, PNAS 112 (36) (2015), p. 11264.

[5] R Danev et al, PNAS 111(44) (2014), p. 15635.

[6] M Schaffer et al, Microsc. Microanal. 21 (Suppl 3) (2015), p. 1119.

[7] J Mahamid et al, Science, 2016, accepted.

[8] S Asano et al, J Mol Biol. 428 (2016), p. 332.

[9] This work was supported by the European Commission grant agreement ERC-2012-SyG_318987ToPAG, the Deutsche Forschungsgemeinschaft Excellence Clusters CIPSM and SFB 1035, and the Max Planck Society.
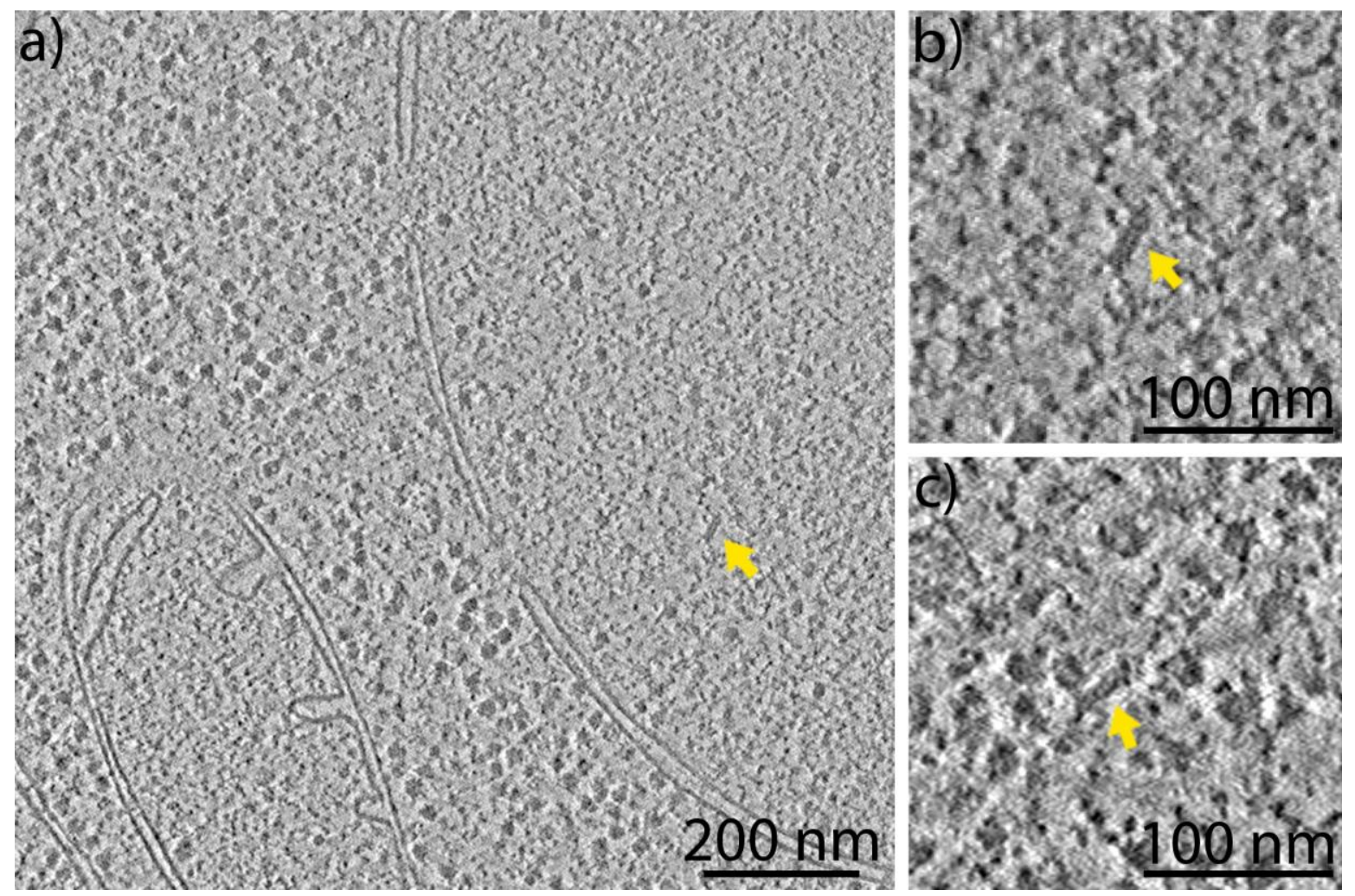

Figure 1. (a) Slice from a reconstructed tomogram of a yeast (Saccharomyces cerevisiae) cell showing the nucleus and mitochondria. Side views of $26 \mathrm{~S}$ proteasomes within the nucleus (b) and in the cytoplasm (c). 